\title{
Limit theorems for the Mellin transform of the square of the Riemann zeta-function. I
}

by

\section{ANTANAS LAURINČIKAS (Vilnius)}

1. Introduction. Let $\mathbb{N}, \mathbb{Z}, \mathbb{R}$ and $\mathbb{C}$ be the sets of all positive integers, integers, real and complex numbers, respectively, and let $s=\sigma+i t$ be a complex variable. The Mellin transform $F(s)$ of the function $f(x)$ is defined by

$$
F(s)=M(f(x))=\int_{0}^{\infty} f(x) x^{s-1} d x .
$$

It is well known that Mellin transforms play an important role in analytic number theory. The study of $F(s)$ is usually easier than that of $f(x)$, and then the inverse formula offers a possibility for the investigation of $f(x)$. Suppose that $f(x)$ is of bounded variation on every finite interval and $f(x) x^{\sigma-1} \in L_{1}(0, \infty)$. Then the inverse formula for $F(s)$ is of the form

$$
\frac{f(x+0)+f(x-0)}{2}=\frac{1}{2 \pi i} \int_{\sigma-i \infty}^{\sigma+i \infty} F(s) x^{-s} d s=\frac{1}{2 \pi i} \lim _{T \rightarrow \infty} \int_{\sigma-i T}^{\sigma+i T} F(s) x^{-s} d s .
$$

Mellin transforms are especially useful for the study of power moments of zeta-functions. Moreover, for this usually some modification of Mellin transforms is applied. The modified Mellin transform $\widetilde{F}(s)$ of the function $f(x)$ is defined by

$$
\widetilde{F}(s)=\widetilde{M}(f(x))=\int_{1}^{\infty} f(x) x^{-s} d x .
$$

This transform is more convenient, since the convergence problem at the point $x=0$ does not arise.

Define

$$
\widehat{f}(x)= \begin{cases}f(1 / x) & \text { if } 0<x \leq 1 \\ 0 & \text { otherwise }\end{cases}
$$


Then in [6] it was observed that

$$
\widetilde{M}(f(x))=M\left(\frac{1}{x} \widehat{f}(x)\right),
$$

and therefore, the properties of the modified Mellin transforms follow from those of the ordinary Mellin transforms.

As noted above, the use of modified Mellin transforms in analytic number theory is primarily related to the study of power moments

$$
I_{k}(T)=\int_{0}^{T}|\zeta(1 / 2+i t)|^{2 k} d t, \quad k \in \mathbb{N},
$$

of the Riemann zeta-function. Define, for some $\sigma>\sigma_{0}(k)$,

$$
\mathcal{Z}_{k}(s)=\int_{1}^{\infty}|\zeta(1 / 2+i x)|^{2 k} x^{-s} d x
$$

Y. Motohashi was the first who observed the usefulness of $\mathcal{Z}_{k}(s)$ in the theory of the Riemann zeta-function. In [13], [14] he introduced and studied the function $\mathcal{Z}_{2}(s)$. The relation between $I_{k}(T)$ and $\mathcal{Z}_{k}(s)$ follows from the Mellin inverse formula: if $f(x)$ is a sufficiently good function, then the equality

$$
\int_{1}^{\infty} f(x / T)|\zeta(1 / 2+i x)|^{2 k} d x=\frac{1}{2 \pi i} \int_{c-i \infty}^{c+i \infty} F(s) T^{s} \mathcal{Z}_{k}(s) d s
$$

with suitable $c>1$ can be used to obtain some information on $I_{k}(T)$.

The investigations of the function $\mathcal{Z}_{2}(s)$ and its applications were continued in [5], [8], [7] and in [10]. In [11] we proved limit theorems in the sense of weak convergence of probability measures for $\mathcal{Z}_{2}(s)$. The aim of this paper is to prove a limit theorem on the complex plane $\mathbb{C}$ for the function $\mathcal{Z}_{1}(s)$.

Note that analytical properties of the functions $\mathcal{Z}_{2}(s)$ and $\mathcal{Z}_{1}(s)$ are quite different. The first results for the function $\mathcal{Z}_{1}(s)$ were obtained in [8]. The definition of $\mathcal{Z}_{1}(s)$ and the asymptotic formula for $I_{1}(T)$ show that the integral defining $\mathcal{Z}_{1}(s)$ converges absolutely for $\sigma>1$, thus the function $\mathcal{Z}_{1}(s)$ is analytic in the half-plane $\{s \in \mathbb{C}: \sigma>1\}$. In [8] the function $\mathcal{Z}_{1}(s)$ has been meromorphically continued to the region $\{s \in \mathbb{C}: \sigma>-3 / 4\}$. The point $s=1$ is its pole of order two with residue $2 \gamma_{0}-\log 2 \pi$, where $\gamma_{0}$ is the Euler constant. M. Jutila proved in [9] that the function $\mathcal{Z}_{1}(s)$ is meromorphically continuable to the whole complex plane with possible poles of order at most two at the points $s=-m, m \in \mathbb{N}$, in the region $\{s \in \mathbb{C}: \sigma<0\}$. Finally, M. Lukkarinen showed in [12] that $\mathcal{Z}_{1}(s)$ in the region $\{s \in \mathbb{C}: \sigma<0\}$ has simple poles only at $s=-(2 m-1), m \in \mathbb{N}$, and she found the formulas for the residues at these points. 
The paper [8] also contains an estimate as well as a mean-square estimate for $\mathcal{Z}_{1}(s)$, namely, for $0 \leq \sigma \leq 1, t \geq t_{0}>0$ and $T \geq 1$,

$$
\mathcal{Z}_{1}(\sigma+i t) \ll_{\varepsilon} t^{1-\sigma+\varepsilon}
$$

and

$$
\int_{1}^{T}\left|\mathcal{Z}_{1}(\sigma+i t)\right|^{2} d t \ll_{\varepsilon} \begin{cases}T^{3-4 \sigma+\varepsilon} & \text { if } 0 \leq \sigma \leq 1 / 2, \\ T^{2-2 \sigma+\varepsilon} & \text { if } 1 / 2 \leq \sigma \leq 1 .\end{cases}
$$

M. Jutila in [9] showed that, for $|s-1| \gg 1$,

$$
\mathcal{Z}_{1}(\sigma+i t) \ll \begin{cases}(|t|+1)^{1-4 \sigma / 3+\varepsilon} & \text { if } 0 \leq \sigma \leq 1 / 2, \\ (|t|+1)^{5 / 6-\sigma+\varepsilon} & \text { if } 1 / 2 \leq \sigma \leq 1\end{cases}
$$

Moreover, M. Lukkarinen proved in [12], for $0 \leq \sigma \leq 1$ and $|t| \geq 2$, that

$$
\mathcal{Z}_{1}(\sigma+i t) \ll|t|^{1-\sigma} \log ^{2}|t|,
$$

which improves (1) and (3) for small values of $\sigma$.

Denote by meas $\{A\}$ the Lebesgue measure of a measurable set $A \subset \mathbb{R}$, and let, for $T>0$,

$$
\nu_{T}(\ldots)=\frac{1}{T} \operatorname{meas}\{t \in[0, T]: \ldots\},
$$

where in place of the dots a condition satisfied by $t$ is to be written. Denote by $\mathcal{B}(S)$ the class of Borel sets of the space $S$.

The probabilistic approach in the investigation of value distribution of the Riemann zeta-function has been proposed by $\mathrm{H}$. Bohr and realized in his joint works with B. Jessen [2], [3]. Later A. Wintner, A. Selberg, A. Ghosh, D. Joyner, B. Bagchi, K. Matsumoto, J. Steuding, E. Stankus, P. D. T. A. Elliott, the present author and others continued and generalized Bohr-Jessen's investigations. However, all these works are related to Dirichlet series, while [11] and this paper deal with integrals. We will prove the following limit theorem of Bohr-Jessen's type.

Theorem 1. Let $\sigma>1 / 2$. Then on $(\mathbb{C}, \mathcal{B}(\mathbb{C}))$ there exists a probability measure $P_{\sigma}$ such that the probability measure

$$
P_{T, \sigma}(A):=\nu_{T}\left(\mathcal{Z}_{1}(\sigma+i t) \in A\right), \quad A \in \mathcal{B}(\mathbb{C}),
$$

converges weakly to $P_{\sigma}$ as $T \rightarrow \infty$.

The function $\mathcal{Z}_{1}(s)$ has a double pole at $s=1$, however in Theorem 1 without loss of generality we can suppose that $\mathcal{Z}_{1}(s)$ is regular for $\sigma>1 / 2$. 
Indeed, let $T_{0}>0$ be fixed. Then

$$
\begin{aligned}
\nu_{T}\left(\mathcal{Z}_{1}(\sigma+i t) \in A\right)= & \frac{1}{T} \operatorname{meas}\left\{t \in\left[0, T_{0}\right]: \mathcal{Z}_{1}(\sigma+i t) \in A\right\} \\
& +\frac{1}{T} \operatorname{meas}\left\{t \in\left[T_{0}, T\right]: \mathcal{Z}_{1}(\sigma+i t) \in A\right\} \\
= & \frac{T-T_{0}}{\left(T-T_{0}\right) T} \operatorname{meas}\left\{t \in\left[T_{0}, T\right]: \mathcal{Z}_{1}(\sigma+i t) \in A\right\}+o(1) \\
= & \frac{1}{T-T_{0}} \operatorname{meas}\left\{t \in\left[T_{0}, T\right]: \mathcal{Z}_{1}(\sigma+i t) \in A\right\}+o(1)
\end{aligned}
$$

uniformly in $A \in \mathcal{B}(\mathbb{C})$. Therefore, instead of the weak convergence of the probability measure $P_{T, \sigma}$ we can investigate that of the probability measure

$$
\frac{1}{T-T_{0}} \operatorname{meas}\left\{t \in\left[T_{0}, T\right]: \mathcal{Z}_{1}(\sigma+i t) \in A\right\}, \quad A \in \mathcal{B}(\mathbb{C}) .
$$

2. A limit theorem for integrals over a finite interval. Let $a>1$, $g(x)$ be an integrable function on $[1, a]$, and

$$
\mathcal{Z}_{g, a}(s)=\int_{1}^{a} g(x) x^{-s} d x .
$$

In this section we will prove limit theorems for the function $\mathcal{Z}_{g, a}(s)$.

TheOREM 2. There exists a probability measure $P_{\sigma, a}$ on $(\mathbb{C}, \mathcal{B}(\mathbb{C}))$ such that the probability measure

$$
P_{T, \sigma, a, g}(A):=\nu_{T}\left(\mathcal{Z}_{g, a}(\sigma+i t) \in A\right), \quad A \in \mathcal{B}(\mathbb{C}),
$$

converges weakly to $P_{\sigma, a}$ as $T \rightarrow \infty$.

The proof of Theorem 2 is based on the following lemma. Denote by $\gamma=\{s \in \mathbb{C}:|s|=1\}$ the unit circle on the complex plane, and define

$$
\Omega_{a}=\prod_{u \in[1, a]} \gamma_{u}
$$

where $\gamma_{u}=\gamma$ for each $u \in[1, a]$. With the product topology and pointwise multiplication $\Omega_{a}$ is a compact topological Abelian group.

Lemma 3. On $\left(\Omega_{a}, \mathcal{B}\left(\Omega_{a}\right)\right)$ there exists a probability measure $Q_{a}$ such that the probability measure

$$
Q_{T, a}(A)=\nu_{T}\left(\left\{u^{i t}: u \in[1, a]\right\} \in A\right), \quad A \in \mathcal{B}\left(\Omega_{a}\right),
$$

converges weakly to $Q_{a}$ as $T \rightarrow \infty$.

Proof. The dual group of $\Omega_{a}$ is

$$
\bigoplus_{u \in[1, a]} \mathbb{Z}_{u}
$$


where $\mathbb{Z}_{u}=\mathbb{Z}$ for each $u \in[1, a]$. The element $\underline{k}=\left\{k_{u}: u \in[1, a]\right\} \in$ $\bigoplus_{u \in[1, a]} \mathbb{Z}_{u}$, where only a finite number of integers $k_{u}$ are distinct from zero, acts on $\Omega_{a}$ by

$$
\underline{x} \rightarrow \underline{x}^{\underline{k}}=\prod_{u \in[1, a]} x_{u}^{k_{u}},
$$

where $\underline{x}=\left\{x_{u}: x_{u} \in \gamma, u \in[1, a]\right\}$. Therefore, the Fourier transform $g_{T, a}(\underline{k})$ of the measure $Q_{T, a}$ is

$$
\begin{aligned}
g_{T, a}(\underline{k}) & =\int_{\Omega_{a}}\left(\prod_{u \in[1, a]} x_{u}^{k_{u}}\right) d Q_{T, a}=\frac{1}{T} \int_{0}^{T}\left(\prod_{u \in[1, a]} u^{i t k_{u}}\right) d t \\
& =\frac{1}{T} \int_{0}^{T} \exp \left\{i t \sum_{u \in[1, a]} k_{u} \log u\right\} d t \\
& = \begin{cases}1 & \text { if } \sum_{u \in[1, a]} k_{u} \log u=0, \\
\frac{\exp \left\{i T \sum_{u \in[1, a]} k_{u} \log u\right\}-1}{i T \sum_{u \in[1, a]} k_{u} \log u} & \text { if } \sum_{u \in[1, a]} k_{u} \log u \neq 0 .\end{cases}
\end{aligned}
$$

Hence we obtain

$$
\lim _{T \rightarrow \infty} g_{T, a}(\underline{k})= \begin{cases}1 & \text { if } \sum_{u \in[1, a]} k_{u} \log u=0, \\ 0 & \text { if } \sum_{u \in[1, a]} k_{u} \log u \neq 0 .\end{cases}
$$

Now the theory of probability measures on locally compact groups (see, for example, [4]) shows that $Q_{T, a}$ converges weakly to the probability measure $Q_{a}$ on $\left(\Omega_{a}, \mathcal{B}\left(\Omega_{a}\right)\right)$ defined by the Fourier transform

$$
g_{a}(\underline{k})= \begin{cases}1 & \text { if } \sum_{u \in[1, a]} k_{u} \log u=0 \\ 0 & \text { if } \sum_{u \in[1, a]} k_{u} \log u \neq 0\end{cases}
$$

as $T \rightarrow \infty$. The lemma is proved.

Proof of Theorem 2. Define a function $h: \Omega_{a} \rightarrow \mathbb{C}$ by the formula

$$
h\left(\left\{y_{x}\right\}\right)=\int_{1}^{a} g(x) x^{-\sigma} y_{x}^{-1} d x, \quad\left\{y_{x}\right\} \in \Omega_{a} .
$$

Then $h$ is continuous, and

$$
h\left(\left\{x^{i t}\right\}\right)=\int_{1}^{a} g(x) x^{-\sigma-i t} d x=\mathcal{Z}_{g, a}(\sigma+i t) .
$$

Hence from Theorem 5.1 of [1] and Lemma 3 we find that the probability measure $P_{T, \sigma, a, g}=Q_{T, a} h^{-1}$ converges weakly to the measure $Q_{a} h^{-1}$ as $T \rightarrow \infty$. 
3. Approximation of the function $\mathcal{Z}_{1}(s)$. Let $\sigma_{1}>1 / 2$ be fixed, and let, for $y \geq 1$,

$$
l_{y}(s)=\frac{s}{\sigma_{1}} \Gamma\left(\frac{s}{\sigma_{1}}\right) y^{s} .
$$

For $\sigma>1 / 2$, define

$$
\mathcal{Z}_{1, y}(s)=\frac{1}{2 \pi i} \int_{\sigma_{1}-i \infty}^{\sigma_{1}+i \infty} \mathcal{Z}_{1}(s+z) l_{y}(z) \frac{d z}{z}
$$

We have $\sigma+\sigma_{1}>1$, therefore, for $\operatorname{Re} z=\sigma_{1}$, the function $\mathcal{Z}_{1}(s+z)$ is represented by the absolutely convergent integral

$$
\mathcal{Z}_{1}(s+z)=\int_{1}^{\infty}|\zeta(1 / 2+i x)|^{2} x^{-(s+z)} d x
$$

Now define

$$
b_{y}(x)=\frac{1}{2 \pi i} \int_{\sigma_{1}-i \infty}^{\sigma_{1}+i \infty}|\zeta(1 / 2+i x)|^{2} \frac{l_{y}(z) d z}{z x^{z}} .
$$

By the well known estimates for the gamma-function we find that

$$
\begin{aligned}
b_{y}(x) & \ll x^{-\sigma_{1}}|\zeta(1 / 2+i x)|^{2} \int_{-\infty}^{\infty}\left|l_{y}\left(\sigma_{1}+i t\right)\right|\left|\sigma_{1}+i t\right|^{-1} d t \\
& \ll_{y} x^{-\sigma_{1}}|\zeta(1 / 2+i x)|^{2} .
\end{aligned}
$$

This together with the estimate

$$
\int_{1}^{T}|\zeta(1 / 2+i t)|^{2} d t \ll T \log T
$$

shows that the integral $\int_{1}^{\infty} b_{y}(x) x^{-s} d x$ converges absolutely for $\sigma>1 / 2$. Therefore, an interchange of order of integration yields

(4) $\int_{1}^{\infty} b_{y}(x) x^{-s} d x=\frac{1}{2 \pi i} \int_{\sigma_{1}-i \infty}^{\sigma_{1}+i \infty}\left(\frac{l_{y}(z)}{z} \int_{1}^{\infty}|\zeta(1 / 2+i x)|^{2} \frac{d x}{x^{s+z}}\right) d z=\mathcal{Z}_{1, y}(s)$.

Let

$$
v(x, y)=\exp \left\{-(x / y)^{\sigma_{1}}\right\}
$$

Then Mellin's formula

$$
\frac{1}{2 \pi i} \int_{b-i \infty}^{b+i \infty} \Gamma(s) c^{-s} d s=e^{-c}
$$

with positive constants $b$ and $c$ shows that

$$
b_{y}(x)=|\zeta(1 / 2+i x)|^{2} v(x, y) .
$$


This and (4) give the formula

$$
\mathcal{Z}_{1, y}(s)=\int_{1}^{\infty}|\zeta(1 / 2+i x)|^{2} v(x, y) x^{-s} d x,
$$

the integral being absolutely convergent for $\sigma>1 / 2$.

Theorem 4. Let $\sigma>1 / 2$ be fixed. Then

$$
\lim _{y \rightarrow \infty} \limsup _{T \rightarrow \infty} \frac{1}{T} \int_{0}^{T}\left|\mathcal{Z}_{1}(\sigma+i t)-\mathcal{Z}_{1, y}(\sigma+i t)\right| d t=0 .
$$

Proof. Clearly, it suffices to consider the case $1 / 2<\sigma<5 / 4$ only, because the integral defining $\mathcal{Z}_{1}(s)$ converges absolutely for $\sigma>1$ and in this region

$$
\lim _{y \rightarrow \infty} \mathcal{Z}_{1, y}(s)=\mathcal{Z}_{1}(s)
$$

uniformly in $t$.

Let $\varepsilon=\sigma-1 / 2$ and $\sigma_{2}=1 / 2+\varepsilon / 2$. We move the line of integration in the definition of $\mathcal{Z}_{1, y}(s)$ to $\operatorname{Re} z=\sigma_{2}-\sigma$. Between the lines $\operatorname{Re} z=\sigma_{1}$ and $\operatorname{Re} z=\sigma_{2}-\sigma$ the integrand has a simple pole at $z=0$ and a double pole at $z=1-s$. Therefore, the properties of the gamma-function, the estimate (1) and the residue theorem yield

$$
\begin{aligned}
\mathcal{Z}_{1, y}(s)= & \frac{1}{2 \pi i} \int_{\sigma_{2}-\sigma-i \infty}^{\sigma_{2}-\sigma+i \infty} \mathcal{Z}_{1}(s+z) l_{y}(z) \frac{d z}{z} \\
& +\mathcal{Z}_{1}(s)+\operatorname{Re} s_{z=1-s} \mathcal{Z}_{1}(s+z) l_{y}(z) z^{-1}
\end{aligned}
$$

By the Cauchy integral formula,

$$
\mathcal{Z}_{1}(\sigma+i t)-\mathcal{Z}_{1, y}(\sigma+i t) \ll \int_{\varrho}\left|\mathcal{Z}_{1}(z+i t)-\mathcal{Z}_{1, y}(z+i t)\right||d z|,
$$

where $\varrho$ is the circle $|z-\sigma|=\varepsilon / 4$. Hence, for sufficiently large $T$,

$$
\begin{aligned}
\frac{1}{T} \int_{0}^{T} \mid \mathcal{Z}_{1}(\sigma+i t)- & \mathcal{Z}_{1, y}(\sigma+i t) \mid d t \\
& \ll \frac{1}{T} \int_{\varrho}|d z| \int_{0}^{2 T}\left|\mathcal{Z}_{1}(\operatorname{Re} z+i t)-\mathcal{Z}_{1, y}(\operatorname{Re} z+i t)\right| d t \\
& \ll \frac{1}{T} \sup _{\sigma \in \varrho} \int_{0}^{2 T}\left|\mathcal{Z}_{1}(\sigma+i t)-\mathcal{Z}_{1, y}(\sigma+i t)\right| d t
\end{aligned}
$$


Since $\Gamma(s) \ll e^{-c|t|}, c>0$, using (1) we obtain

$$
\frac{1}{T} \int_{0}^{T}|R(\sigma+i t)| d t=o(1)
$$

as $T \rightarrow \infty$, where

$$
R(s)=\operatorname{Re} s_{z=1-s} \mathcal{Z}_{1}(s+z) l_{y}(z) z^{-1} .
$$

Moreover, by (5),

$$
\begin{aligned}
\mathcal{Z}_{1}(\sigma+i t) & -\mathcal{Z}_{1, y}(\sigma+i t) \\
& \ll \int_{-\infty}^{\infty}\left|\mathcal{Z}_{1}\left(\sigma_{2}+i t+i u\right)\right|\left|l_{y}\left(\sigma_{2}-\sigma+i u\right)\right| d u+|R(\sigma+i t)| .
\end{aligned}
$$

From this and (7) we deduce that

$$
\begin{aligned}
\frac{1}{T} \int_{0}^{2 T}\left|\mathcal{Z}_{1}(\sigma+i t)-\mathcal{Z}_{1, y}(\sigma+i t)\right| d t & \\
& \ll \int_{-\infty}^{\infty}\left|l_{y}\left(\sigma_{2}-\sigma+i u\right)\right|\left(\frac{1}{T} \int_{-|u|}^{|u|+2 T}\left|\mathcal{Z}_{1}\left(\sigma_{2}+i t\right)\right| d t\right) d u+o(1)
\end{aligned}
$$

as $T \rightarrow \infty$. The Cauchy-Schwarz inequality and (2) imply the estimate

$$
\int_{0}^{T}\left|\mathcal{Z}_{1}\left(\sigma_{2}+i t\right)\right| d t \ll T .
$$

Therefore, by (8),

$$
\begin{aligned}
\frac{1}{T} \sup _{\substack{\sigma \\
s \in \varrho}} \int_{0}^{2 T}\left|\mathcal{Z}_{1}(\sigma+i t)-\mathcal{Z}_{1, y}(\sigma+i t)\right| d t & \\
& \ll \sup _{\substack{\sigma \in \rho \\
s \in \infty}}^{\infty}\left|l_{y}\left(\sigma_{2}-\sigma+i u\right)\right|(1+|u|) d u \\
& \ll \sup _{\sigma \leq-\varepsilon / 4} \int_{-\infty}^{\infty}\left|l_{y}(\sigma+i u)\right|(1+|u|) d u=o(1)
\end{aligned}
$$

as $y \rightarrow \infty$. This, (8) and (6) prove the theorem.

4. A limit theorem for the function $\mathcal{Z}_{1, y}(s)$. In this section we consider the weak convergence of the probability measure

$$
P_{T, \sigma, y}(A):=\nu_{T}\left(\mathcal{Z}_{1, y}(\sigma+i t) \in A\right), \quad A \in \mathcal{B}(\mathbb{C}) .
$$


TheOREm 5. Let $\sigma>1 / 2$. Then on $(\mathbb{C}, \mathcal{B}(\mathbb{C}))$ there exists a probability measure $P_{\sigma, y}$ such that the measure $P_{T, \sigma, y}$ converges weakly to $P_{\sigma, y}$ as $T \rightarrow \infty$.

Proof. In the definition of the function $\mathcal{Z}_{g, a}(s)$ in Section 2 we put

$$
g_{y}(x)=|\zeta(1 / 2+i x)|^{2} v(x, y),
$$

and denote the resulting function by $\mathcal{Z}_{1, a, y}(s)$. Let $P_{T, \sigma, a, y}$ be the corresponding $\mathcal{Z}_{1, a, y}(s)$ measure in Theorem 2 . Then by Theorem 2 the measure $P_{T, \sigma, a, y}$ converges weakly to some measure $P_{\sigma, a, y}$ on $(\mathbb{C}, \mathcal{B}(\mathbb{C}))$ as $T \rightarrow \infty$. We will prove that the family of probability measures $\left\{P_{\sigma, a, y}\right\}$ is tight for fixed $y$.

Suppose that the random variable $\theta_{T}$ is defined on a certain probability space $(\Omega, \mathcal{B}(\Omega), \mathbb{P})$ and uniformly distributed on the interval $[0, T]$. Define

$$
X_{T, a, y}(\sigma)=\mathcal{Z}_{1, a, y}\left(\sigma+i \theta_{T}\right)
$$

and denote by $\stackrel{\mathcal{D}}{\longrightarrow}$ the convergence in distribution. Then by the above remark

$$
X_{T, a, y}(\sigma) \underset{T \rightarrow \infty}{\stackrel{\mathcal{D}}{\longrightarrow}} X_{a, y}(\sigma)
$$

where $X_{a, y}(\sigma)$ is a complex-valued random variable with the distribution $P_{\sigma, a, y}$. For any $M>0$, the Chebyshev inequality yields

$$
\mathbb{P}\left(\left|X_{T, a, y}(\sigma)\right|>M\right) \leq \frac{1}{T M} \int_{0}^{T}\left|\mathcal{Z}_{1, a, y}(\sigma+i t)\right| d t .
$$

As we have seen in Section 3, the integral defining $\mathcal{Z}_{1, y}(s)$ converges absolutely for $\sigma>1 / 2$. Therefore,

$$
\sup _{a \geq 1} \limsup _{T \rightarrow \infty} \frac{1}{T} \int_{0}^{T}\left|\mathcal{Z}_{1, a, y}(\sigma+i t)\right| d t \leq R<\infty .
$$

Now we take $M=R \varepsilon^{-1}$, where $\varepsilon$ is an arbitrary positive number. Then from (10) and (11) we obtain

$$
\limsup _{T \rightarrow \infty} \mathbb{P}\left(\left|X_{T, a, y}(\sigma)\right|>M\right) \leq \varepsilon .
$$

The function $h: \mathbb{C} \rightarrow \mathbb{R}$ given by $h(s)=|s|$ is continuous, therefore (9) and Theorem 5.1 of [1] show that

Therefore, by (12),

$$
\left|X_{T, a, y}(\sigma)\right| \underset{T \rightarrow \infty}{\stackrel{\mathcal{D}}{\longrightarrow}}\left|X_{a, y}(\sigma)\right| .
$$

$$
\mathbb{P}\left(\left|X_{a, y}(\sigma)\right|>M\right) \leq \varepsilon .
$$

The set $K_{\varepsilon}=\{s \in \mathbb{C}:|s| \leq M\}$ is compact, and by the latter inequality, $\mathbb{P}\left(\left|X_{a, y}(\sigma)\right| \in K_{\varepsilon}\right) \geq 1-\varepsilon$ for all $a>1$. This and the definition of the 
random variable $X_{a, y}(\sigma)$ show that $P_{\sigma, a, y}\left(K_{\varepsilon}\right) \geq 1-\varepsilon$ for all $a>1$, i.e. the family of probability measures $\left\{P_{\sigma, a, y}\right\}$ is tight. Hence by the Prokhorov theorem (see, for example, [1]), it is relatively compact.

Since, for $\sigma>1 / 2$,

$$
\lim _{a \rightarrow \infty} \mathcal{Z}_{1, a, y}(s)=\mathcal{Z}_{1, y}(s)
$$

we deduce that, for every $\varepsilon>0$,

$$
\begin{aligned}
& \lim _{a \rightarrow \infty} \limsup _{T \rightarrow \infty} \nu_{T}\left(\left|\mathcal{Z}_{1, a, y}(\sigma+i t)-\mathcal{Z}_{1, y}(\sigma+i t)\right| \geq \varepsilon\right) \\
& \leq \lim _{a \rightarrow \infty} \limsup _{T \rightarrow \infty} \frac{1}{\varepsilon T} \int_{0}^{T}\left|\mathcal{Z}_{1, a, y}(\sigma+i t)-\mathcal{Z}_{1, y}(\sigma+i t)\right| d t=0 .
\end{aligned}
$$

Setting $X_{T, y}(\sigma)=\mathcal{Z}_{1, y}\left(\sigma+i \theta_{T}\right)$, we hence find that

$$
\lim _{a \rightarrow \infty} \limsup _{T \rightarrow \infty} \mathbb{P}\left(\left|X_{T, a, y}(\sigma)-X_{T, y}(\sigma)\right| \geq \varepsilon\right)=0 .
$$

The relative compactness of $\left\{P_{\sigma, a, y}\right\}$ shows that there exists a subsequence $P_{\sigma, a_{1}, y} \subset\left\{P_{\sigma, a, y}\right\}$ such that $P_{\sigma, a_{1}, y}$ converges weakly to some probability measure $P_{\sigma, y}$ on $(\mathbb{C}, \mathcal{B}(\mathbb{C}))$ as $a_{1} \rightarrow \infty$. Thus

$$
X_{a_{1}, y}(\sigma) \underset{a_{1} \rightarrow \infty}{\stackrel{\mathcal{D}}{\longrightarrow}} P_{\sigma, y} .
$$

This, (9) and (13) show that all hypotheses of Theorem 4.2 of [1] are satisfied, therefore

$$
X_{T, y}(\sigma) \underset{T \rightarrow \infty}{\stackrel{\mathcal{D}}{\longrightarrow}} P_{\sigma, y}
$$

and the theorem is proved.

5. Proof of Theorem 1. Theorem 1 follows from Theorem 5 in the same way as Theorem 5 from Theorem 2 . We preserve the notation of the previous section.

First we show that the family $\left\{P_{\sigma, y}\right\}$ of probability measures, where $P_{\sigma, y}$ is the limit measure in Theorem 5, is tight. By Theorem 5,

$$
X_{T, y}(\sigma) \underset{T \rightarrow \infty}{\stackrel{\mathcal{D}}{\longrightarrow}} X_{y}(\sigma)
$$

where $X_{y}(\sigma)$ is a complex-valued random variable having the distribution $P_{\sigma, y}$. Define $R$ by

$$
\sup _{y \geq 1} \limsup _{T \rightarrow \infty} \frac{1}{T} \int_{0}^{T}\left|\mathcal{Z}_{1, y}(\sigma+i t)\right| d t \leq R .
$$


Since

$$
\begin{aligned}
\limsup _{T \rightarrow \infty} \frac{1}{T} \int_{0}^{T}\left|\mathcal{Z}_{1, y}(\sigma+i t)\right| d t & \ll \limsup _{T \rightarrow \infty}\left(\frac{1}{T} \int_{0}^{T}\left|\mathcal{Z}_{1, y}(\sigma+i t)\right|^{2} d t\right)^{1 / 2} \\
& \ll\left(\int_{1}^{\infty}|\zeta(1 / 2+i x)|^{4} x^{-2 \sigma} v^{2}(x, y) d x\right)^{1 / 2}<\infty,
\end{aligned}
$$

we have $R<\infty$, and taking $M=R \varepsilon^{-1}$, we deduce from (14), similarly to the proof of Theorem 5 , that $P_{\sigma, y}\left(K_{\varepsilon}\right) \geq 1-\varepsilon$ for all $y \geq 1$. This proves the tightness of $\left\{P_{\sigma, y}\right\}$. Hence $\left\{P_{\sigma, y}\right\}$ is relatively compact.

Theorem 4 and the Chebyshev inequality show that, for every $\varepsilon>0$,

$$
\lim _{y \rightarrow \infty} \limsup _{T \rightarrow \infty} \nu_{T}\left(\left|\mathcal{Z}_{1}(\sigma+i t)-\mathcal{Z}_{1, y}(\sigma+i t)\right| \geq \varepsilon\right)=0 .
$$

Now let $X_{T}(\sigma)=\mathcal{Z}_{1}\left(\sigma+i \theta_{T}\right)$. Then (15) implies

$$
\lim _{y \rightarrow \infty} \limsup _{T \rightarrow \infty} \mathbb{P}\left(\left|X_{T, y}(\sigma)-X_{T}(\sigma)\right| \geq \varepsilon\right)=0 .
$$

We choose $\left\{P_{\sigma, y_{1}}\right\} \subset\left\{P_{\sigma, y}\right\}$ such that $P_{\sigma, y_{1}}$ converges weakly to some probability measure $P_{\sigma}$ on $(\mathbb{C}, \mathcal{B}(\mathbb{C}))$ as $y_{1} \rightarrow \infty$. Then

$$
X_{y_{1}}(\sigma) \underset{y_{1} \rightarrow \infty}{\stackrel{\mathcal{D}}{\longrightarrow}} P_{\sigma} .
$$

This, (14), (16) and Theorem 4.2 of [1] prove the theorem.

\section{References}

[1] P. Billingsley, Convergence of Probability Measures, Wiley, New York, 1968.

[2] H. Bohr und B. Jessen, Über die Wertverteilung der Riemannschen Zetafunktion, Erste Mitteilung, Acta Math. 54 (1930), 1-35.

[3] -, - Über die Wertverteilung der Riemannschen Zetafunktion, Zweite Mitteilung, ibid. 58 (1932), 1-55.

[4] H. Heyer, Probability Measures on Locally Compact Groups, Springer, Berlin, 1977.

[5] A. Ivić, The Mellin transform and the Riemann zeta-function, in: Proc. Conf. on Elementary and Analytic Number Theory (Vienna, 1996), W. G. Nowak and J. Schoißengeier (eds.), Univ. Wien \& Univ. für Bodenkultur, Vienna, 1996, 112127.

[6] - On some conjectures and results for the Riemann zeta-function and Hecke series, Acta Arith. 99 (2001), 115-145.

[7] - On the estimation of $\mathcal{Z}_{2}(s)$, in: Analytic and Probabilistic Methods in Number Theory (Palanga, 2001), A. Dubickas et al. (eds.), TEV, Vilnius, 2002, 83-98.

[8] A. Ivić, M. Jutila and Y. Motohashi, The Mellin transform of powers of the zetafunction, Acta Arith. 95 (2000), 305-342.

[9] M. Jutila, The Mellin transform of the square of Riemann's zeta-function, Period. Math. Hungar. 42 (2001), 179-190.

[10] - The Mellin transform of the fourth power of Riemann's zeta- function, in: Proc. Conf. on Analytic Number Theory with Special Emphasis on $L$-functions, Inst. Math. Sci., Chennai, 2004, 15-29. 
[11] A. Laurinčikas, Limit theorems for the Mellin transform of $|\zeta(1 / 2+i t)|^{4}$, preprint, Vilnius Univ., 2005.

[12] M. Lukkarinen, The Mellin transform of the square of Riemann's zeta-function and Atkinson's formula, Ann. Acad. Sci. Fenn. Math. Diss. 140 (2005).

[13] Y. Motohashi, A relation between the Riemann zeta-function and the hyperbolic Laplacian, Ann. Scuola Norm. Sup. Pisa Cl. Sci. (4) 22 (1995), 299-313.

[14] -, Spectral Theory of the Riemann Zeta-Function, Cambridge Univ. Press, Cambridge, 1997.

Department of Mathematics and Informatics

Vilnius University

Naugarduko 24

03225 Vilnius, Lithuania

E-mail: antanas.laurincikas@maf.vu.lt

Received on 13.4.2005

and in revised form on 6.1.2006 